\title{
Molecular First Hyperpolarizabilities of a New Class of Asymmetric Squaraine Dyes
}

\author{
Chin-Ti Chen, ${ }^{a}$ Seth R. Marder, ${ }^{*}$ ab and Lap-Tak Cheng ${ }^{c}$ \\ a Molecular Materials Resource Center, The Beckman Institute, 139-74, California Institute of Technology, Pasadena, CA, \\ 91125, USA \\ b Jet Propulsion Laboratory, California Institute of Technology, 4800 Oak Grove Drive, Pasadena, CA 91109, USA \\ c Central Research and Engineering Laboratories, E. I. DuPont de Nemours and Company, Inc., Wilmington, DE \\ 19880-0356, USA
}

The molecular first hyperpolarizabilities $(\beta)$ of a series of asymmetric squaraine dyes have been measured by electric-field-induced second harmonic generation; the dyes have negative $\beta$-values whose magnitudes are comparable to 4-N,N-dimethylamino-4'-nitrostilbene (DANS).

Bond length alternation (BLA) (i.e. the difference between average length of carbon-carbon double and single bonds in a polymethine chain) is a useful parameter to consider when establishing structure-property relationships for nonlinear optical (NLO) molecules. ${ }^{1-5}$ Electric field dependent calculations on $(\mathrm{Me})_{2} \mathrm{~N}-(\mathrm{CH}=\mathrm{CH})_{4}-\mathrm{CHO}$ show that with increasing applied electric field the geometry of the molecule changes from the neutral polyene structure (BLA $\geqslant-0.12 \AA$ ) (on the left in Fig. 1) to the polar cyanine structure (BLA $=0 \AA)$ and to a highly polar zwitterionic polyene structure (BLA ca. 0.10 $\AA$ ). At the same time the molecular first hyperpolarizability $(\beta)$ initially increases, reaches a peak at roughly BLA ca. $-0.04 \AA$ (closer to the cyanine limit than to the polyene limit), decreases and ultimately becomes negative when BLA is positive. Most donor-acceptor substituted $\pi$-conjugated molecules, such as stilbenes and polyenes, have positive $\beta$-values that increase as the strength of donors and acceptors increases. ${ }^{6,7}$ Moreover, these chromophores usually have BLA greater than $-0.10 \AA$ and therefore are far from having maximized $\beta .{ }^{8}$ Rather than starting near the polyene limit and decreasing the magnitude of BLA by searching for stronger donors or acceptors, to optimize $\beta$, we have considered introducing asymmetry into highly polarizable cyanine-like structures as an alternative strategy. In this manner, we seek to optimize $\beta$ (in either a positive or negative sense) starting from the cyanine limit where BLA $=0$. Squaraine dyes are analogous to cyanine dyes in that they both have two degenerate resonance forms (Fig. 2) and exhibit sharp, intense absorption bands in the visible or near-IR region. ${ }^{9}$ However, unlike ionic cyanines, squaraines are neutral molecules that are suitable for electric-field-induced second harmonic generation (EFISH) measurements. $\dagger$ Previously, it was demonstrated that symmetric squaraines have large molecular second hyperpolarizabilities $(\gamma) \cdot{ }^{10}$

Here, we report the optical nonlinearities of a new series of asymmetric squaraine compounds (Fig. 3). The dyes were prepared by the condensation between appropriate donors,

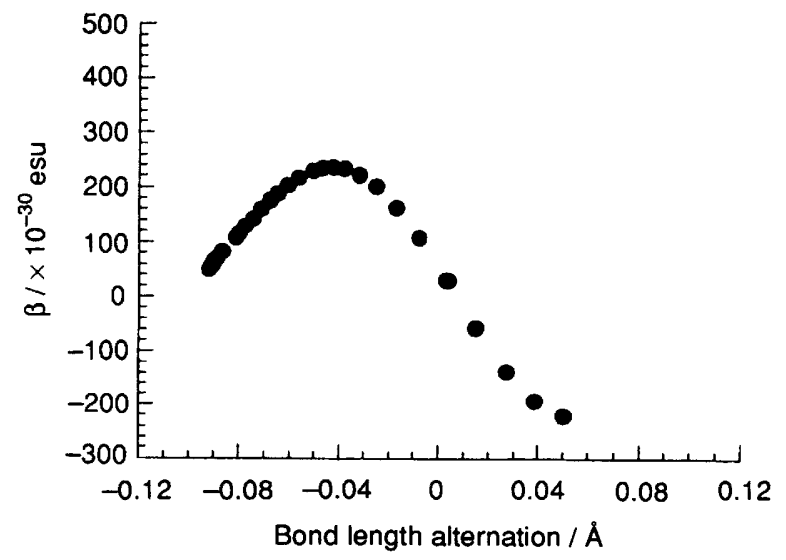

Fig. 1 Plot of $\beta$ vs. bond length alternation (BLA) for $(\mathrm{Me})_{2} \mathrm{~N}$ $(\mathrm{CH}=\mathrm{CH})_{4}$ - $\mathrm{CHO}$. heterocyclic rings with activated methyl groups, and 1-aryl-2hydroxycyclobutene-3,4-diones 1 , in a procedure analogous to that developed by Law et al. ${ }^{11}$ Since asymmetric squaraines tend to decompose on silica or alumina, the success of the syntheses hinges upon being able to impart the correct solubility, such that the products crystallize directly from the crude reaction mixtures. However, the solubility of asymmetric squaraines must, at the same time, be high enough for EFISH studies. Depending on the donors, isopentyloxy and methoxy groups of 1 appear to satisfy these criteria. Analytically pure products were obtained in $30-80 \%$ yield. $\ddagger$ In polar organic solvents, the asymmetric squaraine dyes 2-7 are in general more stable than [4-(dimethylamino)phenyl] $\left(4^{\prime}\right.$ methoxyphenyl)squaraine $\mathbf{8}$ and its derivatives prepared by Law et al. ${ }^{12}$ For instance, the pyridine solution $(\sim 0.1$ mmol dm $\mathrm{dm}^{-3}$ ) of 8 decolourized completely in $1 \mathrm{~h}$, whereas the absorption spectra solutions of $\mathbf{3}$ or $\mathbf{5}$ showed little evidence of deterioration after several days.

EFISH data of $\beta$ (measured in chloroform with $1.907 \mu \mathrm{m}$ fundamental radiation ${ }^{6,12}$ ) and their zero frequency form $\beta_{0}$ (corrected for dispersive enhancement using the two-state model ${ }^{13}$ ) for the asymmetric squaraines 2-7 are shown in Table 1, together with the data of optical absorption maximum
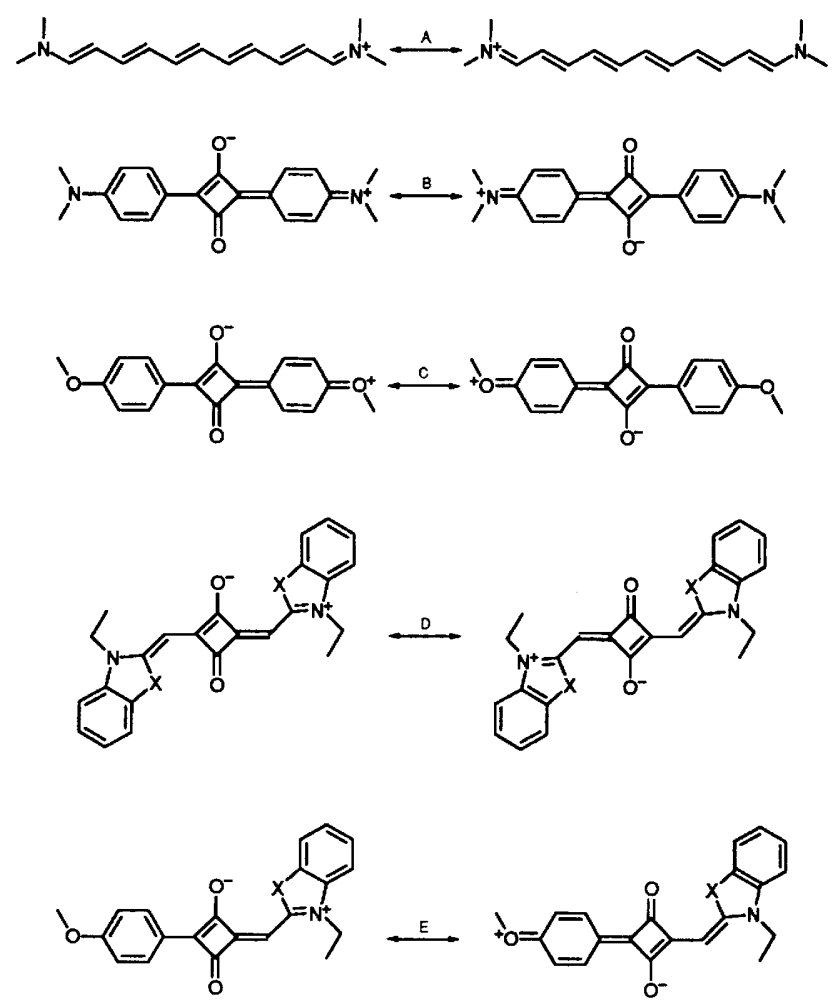

Fig. 2 Canonical resonance structures of cyanine $\mathbf{A}$, squaraines $\mathbf{B}, \mathbf{C}$, and $\mathbf{D}$, and asymmetric squaraines $\mathbf{E}$. X can be $\mathrm{O}, \mathrm{S}, \mathrm{Se}$, or $\mathrm{CMe}_{2}$. 


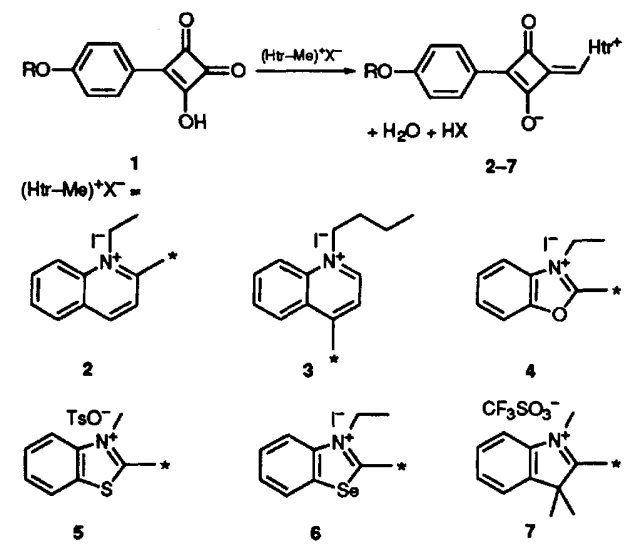

Fig. 3 Synthesis of asymmetric squaraines 2-7. Symbol* indicates the active site of donors that react to 1 . Here $\mathrm{R}$ equals to isopentyl for quinaldinium 2, benzothiazolium 5 and benzoselenazolium 6; methyl for lepidinium 3, benzoxazolium 4 and indolenium 7 . Ts $\mathrm{O}^{-}$denotes toluene-p-sulfonate.

Table 1 Optical data of asymmetric squaraines 2-7 measured in chloroform with $1.907 \mu \mathrm{m}$ as fundamental radiation

\begin{tabular}{llclcl}
\hline & \multicolumn{2}{c}{$\begin{array}{c}\varepsilon / \mathrm{dm}^{3} \mathrm{~mol}^{-1} \\
\mu / 10^{-18}\end{array}$} & $\begin{array}{l}\beta / 10^{-30} \\
\text { esu }\end{array}$ & $\begin{array}{l}\beta_{0} / 10^{-30} \\
\text { esu }\end{array}$ \\
\hline $\mathbf{2}$ & 572 & 6.0 & 7.5 & -94 & -55 \\
$\mathbf{3}$ & 616 & 14 & 8.5 & -120 & -63 \\
$\mathbf{4}$ & 518 & 9.6 & 7.5 & -42 & -27 \\
$\mathbf{5}$ & 554 & 10 & 7.7 & -95 & -58 \\
$\mathbf{6}$ & 562 & 10 & 6.6 & -41 & -24 \\
$\mathbf{7}$ & 556 & 11 & 7.5 & -69 & -42 \\
\hline
\end{tabular}

$\left(\lambda_{\max }\right)$, extinction coefficient $(\varepsilon)$ and the dipole moment $(\mu)$. The $\beta_{0^{-v}}$-values of $2-7$, range from -24 to $-63 \times 10^{-30} \mathrm{esu}$, are comparable to that of the prototypical stilbene compound 4- $N, N$-dimethylamino-4'-nitrostilbene (DANS) $\left(\beta_{0}=55 \times\right.$ $10^{-30}$ esu) but are opposite in sign. The magnitude of the $\beta$-values of 2-7 follows roughly the order of conventional donor strength, i.e. lepidinium $3>$ quinaldinium 2; benzothiazolium $5>$ benzoselenazolium 6 , benzoxazolium 4 and indolenium 7.14 Comparison of $\beta$-values between asymmetric squaraines with donors whose structure differ significantly (e.g. lepidinium and benzothiazolium derivatives), is difficult without the knowledge of the $\mu$ orientation with respect to the $\beta$ tensor since EFISH only measures the projection of the $\beta$ tensor along the dipole direction.

Chromophores with negative $\beta$-values are uncommon. However, two examples are merocyanine and dicyanoquinodial compounds. ${ }^{15}$ The negative $\beta$-values of the dyes studied here are consistent with their negative solvatochromism (e.g. $\lambda_{\max }$ for 3 appear at $562 \mathrm{~nm}$ in ethanol, $616 \mathrm{~nm}$ in chloroform and $664 \mathrm{~nm}$ in toluene). With the concept of the two-state model, 13 the negative $\beta$-values of asymmetric squaraine dyes suggest that the dyes either have a larger ground- than excited-state dipole or the direction of the dipole moment actually changes sign in the excited-state. Thus, one question is whether the intramolecular charge-transfer of asymmetric squaraines involves a shift of the charge from one end of the molecules to the other end or only from one end to the central squaraine ring $\left(\mathrm{C}_{4} \mathrm{O}_{2}\right)$. This is not understood at this stage and we are synthesizing model compounds to probe the question.
This work was performed, in part, by the Center for Space Microelectronics Technology, Jet Propulsion Laboratory (JPL), California Institute of Technology and was sponsored by the Ballistic Missiles Defence Organization, Innovative Science and Technology Office and The Advanced Research Projects Agency through grant \# 91-NC-146 administered by the Air Force Office of Scientific Research through agreements with NASA. Support at the Beckman Institute from the National Science Foundation (Grant No. CHE 9106689) is gratefully acknowledged. We thank Professor Anthanv Garito for helpful discussions.

Received, 1st September 1993; Com. 3105245A

\section{Footnotes}

$\dagger$ The definition of BLA that we have used for polymethine dye does not directly apply to squaraine dyes, as they have no polymethine chain. The idea of desymmetrizing squaraine may nonetheless be analogous to desymmetrizing cyanine dyes.

\$ 2-7 gave ${ }^{1} \mathrm{H}$ NMR, UV-VIS, FABMS, fully consistent with the proposed structure. Analytically pure samples were obtained by recrystallization from pyridine and hexanes. Satisfactory elemental analyses were obtained for all compounds except 4 which tends to decompose in pyridine.

\section{References}

1 S. R. Marder, D. N. Beratan and L.-T. Cheng, Science, 1991, 252 103.

2 B. G. Tiemann, L.-T. Cheng and S. R. Marder, J. Chem. Soc., Chem. Commun., 1993, 735.

3 S. R. Marder, C. B. Gorman, L.-T. Cheng and B. G. Tiemann, Proc. SPIE, 1992, 1775, 19.

4 S. R. Marder, J. W. Perry, G. Bourhill, C. B. Gorman, B. G. Tiemann and K. Mansour, Science, 1993, 261, 186.

5 S. R. Marder, C. B. Gorman, B. G. Tiemann and L.-T. Cheng, J. Am. Chem. Soc., 1993, 115, 3006.

6 L.-T. Cheng, W. Tam, S. H. Stevenson, G. R. Meredith, G. Rikken and S. R. Marder, J. Phys. Chem., 1991, 95, 10631

7 L.-T. Cheng, W. Tam, S. R. Marder, A. E. Stiegman, G. Rikken and C. W. Spangler, J. Phys. Chem., 1991, 95, 10643.

8 S. R. Marder, J. W. Perry, B. G. Tiemann, C. B. Gorman, S. Gilmour, S. L. Biddle and G. Bourhill, J. Am. Chem. Soc., 1993, 115,2524 and references therein.

9 R. W. Bigelow and H. J. Freund, Chem. Phys., 1986, 107, 159, K.-Y. Law, J. Phys. Chem., 1987, 91, 5184; P. A. Cahill and K. D. Singer, in Materials for Nonlinear Optics: Chemical Perspectives, ed. S. R. Marder, J. E. Sohn and G. D. Stucky, ACS Symp. Ser., No. 455 ACS, Washington DC, 1991, p. 200.

10 C. W. Dirk and M. G. Kuzyk, Chem. Mater., 1990, 2, 4; C. W. Dirk and M. G. Kuzyk, in Materials for Nonlinear Optics: Chemical Perspectives, ed. S. R. Marder, J. E. Sohn and G. D. Stucky, ACS Symp. Ser., No. 455, ACS, Washington DC, 1991, p. 687; C. W. Dirk, L.-T. Cheng and M. G. Kuzyk, Int. J. Quant. Chem. 1992, 43, 27; C. W. Dirk, N. Caballero, A. Tan, M. G. Kuzyk, L.-T. Cheng, H. Katz, M. Schilling and L. King, Proc. SPIE, 1992, 1775, 64 .

11 K.-Y. Law and F. C. Bailey, J. Org. Chem., 1992, 57, 3278.

12 B. F. Levine and C. G. Bethea, Appl. Phys. Lett., 1974, 24, 445.

13 J. L. Oudar and D. S. Chemla, J. Chem. Phys., 1977, 66, 2664 J. L. Oudar, J. Chem. Phys., 1977, 67, 446.

$14 \mathrm{~J}$. Fabian and H. Hartmann, Light Absorption of Organic Colorants, Springer, Berlin, 1980, p. 179.

15 P. N. Prasad and D. J. Williams, Introduction to Nonlinear Optical Effects in Molecules and Polymers, Wiley, NY, 1991, p. 141. 